\title{
Delay in Early Infant Diagnosis and High Loss to Follow-Up among Infant Born to HIV-Infected Women in Ethiopia
}

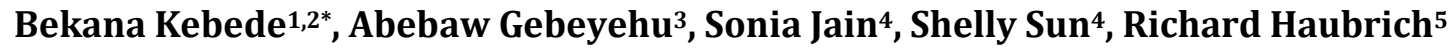 \\ ${ }^{1}$ Antiviral Research Center, School of Medicine, University of California San Diego, San Diego, USA \\ ${ }^{2}$ Department of Health Service Management, Institute of Public Health, University of Gondar, Gondar, Ethiopia \\ ${ }^{3}$ Department of Reproductive Health, Institute of Public Health, University of Gondar, Gondar, Ethiopia \\ ${ }^{4}$ Department of Family and Preventive Medicine, Division of Biostatistics and Bioinformatics, University of \\ California San Diego, San Diego, USA \\ ${ }^{5}$ Division of Infectious Disease, School of Medicine, University of California San Diego, San Diego, USA \\ Email: bekanasiif@gmail.com
}

Received 18 September 2014; revised 15 October 2014; accepted 11 November 2014

Copyright (C) 2014 by authors and Scientific Research Publishing Inc.

This work is licensed under the Creative Commons Attribution International License (CC BY).

http://creativecommons.org/licenses/by/4.0/

c) (i)

Open Access

\section{Abstract}

Background: Many HIV-infected infants and children die from HIV related causes without their HIV status being known or receiving HIV care. All HIV exposed infants should be tested by Dried Blood Spots (DBS)-PCR before or at 6 weeks of age. Testing is a crucial step to facilitate early access to antiretroviral treatment (ART). However, studies that assess the level of use and implementation of HIV DNA testing in Ethiopia are lacking. Objective: To investigate the rate of early infant diagnosis (EID), defined as having blood drawn for HIV DNA-PCR testing, and predictive factors of EID among infants born to HIV infected women. Method: A multicentre retrospective cohort study was conducted from April to June 2012 in three public hospitals and three health centers, in Northwest Ethiopia. Mother-infant pairs were followed from delivery until the time of the HIV diagnostic test. Data were captured using standardized forms. The time-to-diagnostic test was estimated using Kaplan-Meier estimators. Factors associated with EID were evaluated using logistic regression. Result: Of the 266 HIV-exposed infants identified from the health facilities, only $109(41.0 \%)$ infants had early HIV DNA-PCR tests. The median age at the time of HIV diagnostic testing was 60 days $195 \% \mathrm{CI}$ : 47 - 73 days), and the median turnaround time between blood draw for DNA-PCR testing to delivery of a test result to the respective health facility was 36 days (95\% CI: 33 - 40 days). A total of 35 (13.2\%) infants were diagnosed with HIV infection. The predictors of EID were the mother having prenatal care, maternal receipt of ART during pregnancy and place of birth. Conclusion: Three out of five HIV-infected women did not bring their infant for HIV testing during the recommended 6 week interval after birth. Special attention is required for infants born to HIV-infected women who did not

\footnotetext{
*Corresponding author.
}

How to cite this paper: Kebede, B., Gebeyehu, A., Jain, S., Sun, S. and Haubrich, R. (2014) Delay in Early Infant Diagnosis and High Loss to Follow-Up among Infant Born to HIV-Infected Women in Ethiopia. World Journal of AIDS, 4, $402-412$. 
receive ART or delivered at home or a private health facility to ensure early infant diagnosis, reduce loss to follow-up and prevent late initiation of ART for HIV-infected infants.

\title{
Keywords
}

\author{
HIV, Infant, Delay, Loss to Follow-Up, PMTCT, Ethiopia
}

\section{Background}

Worldwide, over 2 million children are infected with HIV, 90\% of whom live in sub-Saharan Africa [1]. In 2011, 92\% of pregnant women were living with HIV resided in sub-Saharan Africa [2]. Many HIV-infected infants and children die from HIV related causes without HIV diagnosis or treatment. Without access to cotrimoxazole prophylaxis, antiretroviral therapy (ART) and supportive care, about a third of infants die in the first year of life and a half by 2 years of age [3]. World Health Organization (WHO) pediatric treatment guidelines recommend ART initiation for all HIV-infected infants under 24 months of age [1]. In order to facilitate ART initiation as soon as possible after HIV infection, the WHO recommends HIV diagnostic testing for all HIV-exposed infants (infants born to HIV-infected mothers) at 4 - 6 weeks of age and to initiate therapy soon thereafter [4] [5].

Globally, only 15\% of HIV-exposed infants access early infant diagnosis (EID; i.e., within 45 days of birth) [6]. Studies showed that about 20\% of HIV-positive infants die before six months and 35\% to $40 \%$ die before 12 months [6] [7]. EID is a crucial step to facilitate access to ART, to improve infants' survival and to augment the benefit of PMTCT programmes [6] [7]. Early initiation of ART was associated with better treatment outcomes and increased survival of children born to HIV infected mothers [8].

The WHO recommends that the HIV exposure status of infants be determined at the first contact with the health system, ideally before six weeks of age. All sites providing PMTCT and follow-up services for HIV-exposed infants must be able to collect samples for production of dried blood spots (DBS) for polymerase chain reaction (PCR) HIV DNA testing, even though the test will likely be processed elsewhere. Diagnosis of HIVinfected infants often occurs too late to allow early initiation of ART in many African countries [8]-[10]. The use of DBS test is one of the most effective strategies for increasing uptake of EID, as it enables sample collection and preparation in rural sites that do not have access to health facilities [11].

Based on the single point estimate, Ethiopia has an estimated HIV prevalence of $2.4 \%$ with more than 1 million people living with HIV in 2010 [12]. The HIV prevalence among women aged 15 - 49 is 1.9 percent and the prevalence increases with age to a peak of 3.7 percent at age 30 - 34 [12]. HIV screening is routinely offered to all women seeking prenatal care, and those who are HIV infected are offered antiretroviral (ARV) prophylaxis when eligible by national guidelines [11]. Although Ethiopian government-sponsored programs are available to provide care for all women, less than 20\% received PMTCT in 2010 [7] [13]. Multiple factors, i.e., not seeking health care and high rate of loss to follow-up, lead to low rates of initiating medication during pregnancy and taking ARV at the time of birth. These issues ultimately undermine the efficacy of PMTCT strategies [14]. According to one study in Ethiopia in 2010, only 48\% of known HIV-exposed infants have received ARV prophylaxis at delivery, and the estimated ARV coverage (received the ARV treatment or prophylaxis) for HIV positive mothers and for their babies was found to be $11.6 \%$ and $8.4 \%$, respectively [15].

The other element of PMTCT programmes is the uptake and adherence or follow-up of exposed infants. The provision of EID was started in Ethiopia under the PMTCT programme by the Ministry of Health and partners. The service is offered at all district hospitals and health centers providing PMTCT services free of charge in more than 300 centers including, government Hospitals and health centers. Unfortunately, free services are available in private health facilities. A testing algorithm for infants under 18 months was developed and implemented by the Federal government based on the WHO recommendation. Accordingly, all HIV exposed infants should be tested by DBS at 6 weeks of age and a confirmatory antibody test at 18 months (if a previous antibody test was negative and continued breastfeeding including the provision of provision of cotrimoxazole prophylaxis, and HIV-specific counseling and support [11] [16].

The Ethiopian 2007/8 National PMTCT Guidelines [11] described prophylaxis interventions including Single dose (Sd) NVP (2 mg/kg) + AZT (4 mg/kg Bid for 7 days) and Single dose (Sd) NVP (2 mg/kg) + AZT (4 
$\mathrm{mg} / \mathrm{kg}$ Bid) for four weeks, for HIV positive women who presented during pregnancy and presenting in labour who have not received any antenatal prophylaxis, respectively, and the best time to initiate ARV prophylaxis for the infant is immediately after delivery or within 12 hours if possible. In addition, cotrimoxazole ( $5 \mathrm{mg} / \mathrm{kg}$ daily) should be initiated at 4 weeks, HIV antibody testing at 9 months and at either 18 months of life or 2 months after cessation of breastfeeding.

The goal of EID is to identify HIV infected infants prior to the development of clinical disease to facilitate treatment and follow-up. For infants who are virologically negative, it provides an opportunity to plan and counsel on appropriate feeding to reduce the risk of infection whilst maintaining adequate nutrition. Throughout Africa, the diagnostic challenge of HIV exposure in infants is being addressed by scaling up virological testing using DBS for DNA-PCR [8]. However, despite this scale up, there are no studies defining the uptake and implementation in Ethiopia.

\section{Methods}

\subsection{Study Design}

This was a multicentre retrospective cohort study, which included 266 eligible mother-infant pairs. The study was supported by review of procedures at health facilities that provide PMTCT, infant diagnosis and clinical care and the regional central laboratory to identify the reasons for delay in DNA-PCR testing.

\subsection{Study Setting}

The study was conducted in Amhara region of Ethiopia in three government hospitals and three health centers: Metema Hospital, Gondar University Hospital, Debark Hospital, Woreta health center, Gondar health center and Addis Zemen health center. In this region, the estimated adult HIV prevalence is $2.2 \%$. Only about $10 \%$ of deliveries occur in health facilities; $90 \%$ occur at home or non-health-related locations. The neonatal mortality rate was 54/1000 live births during the period of the study [12]. Metema and Debark hospitals are currently the only two district Public hospitals in the North Gondar regional area. On average, about 500 deliveries are performed in each hospital per year. Gondar University Hospital is the only referral and teaching hospital in the catchment area of North Gondar; it provides service for about 6 million people and has approximately 2000 deliveries every year. Each of the study health facilities provide full PMTCT services including blood collection for infant diagnosis and ART. All 6 health facilities send the blood samples to the Amhara regional laboratory for the HIV DNA-PCR. DBS samples are transported once weekly to the central laboratory.

\subsection{Data Collection Procedure}

The data collection forms were tested for reliability, content and ease of use on a pilot sample of data. Infant HIV testing status by DNA-PCR was determined by review of the maternity and EID service register at the health centers/clinics or hospitals. Abstracted chart data included: date of birth, place of birth, date of first exposed infant clinic visit, date of blood collection, number of days from blood collection to receipt by the central laboratory, time from laboratory receipt to delivery of results at the clinic and infant follow-up for recipient of test result.

At each health facility, charts from HIV-exposed infants were routinely segregated allowing for ease of identification of eligible candidates. All HIV-exposed infants, who had at least one postpartum health center or hospital visit for HIV DNA-PCR testing, were included in the study. The HIV-exposed infants who had no HIV DBS testing or did not have the result recorded were excluded. We also reviewed respective HIV-infected mothers' charts and abstracted relevant including: maternal socio-demographic, PMTCT interventions (including HAART and prophylaxis) including antenatal care, place of delivery, and maternal health conditions. On the exposed infant record sheet, both the DBS test and HIV antibody test results were recorded. HIV infection in this study was defined by either a positive HIV DNA-PCR test or a positive HIV antibody result recorded on the infant diagnosis chart. Note, Ethiopian national guidelines specify that infant HIV antibody testing should be done after 6 months of age, however, in some cases the data of antibody testing was not recorded in the database (all antibody tests were inferred to have been done according to guidelines). For some missing data in the health record, information was obtained during an in-depth interview (conducted by study personnel) using the case 
report forms. The interview was conducted at the time when women returned to the health facilities for a mother-to-mother support group (MSG) meeting.

Data abstraction was done by trained data collection assistants and the investigator. Data quality and completeness was checked for each data form. All mother/infant pairs were followed from the time of birth until the initial EID visit. Review of standard operating procedures at health facilities that provide PMTCT, infant diagnosis and clinical care and the regional central laboratory center was performed in order to identify the reasons for delay in DNA-PCR testing from the blood draw to the arrival of test result was done.

\subsection{Ethics Statement}

The study was reviewed and approved by the Ethical Review Board of the Institute of Public Health, at the University of Gondar for all the study ethical procedures. Permission was obtained from the medical director of each hospital and head of the health centers. Both written and verbal informed consent was obtained from the next of kin, caretakers, or guardians on behalf of the children enrolled in the study, and participants consent was documented on consent form attached with each questionnaire. Personal information remained confidential.

\subsection{Data Analysis}

Data was coded, entered, cleaned and analyzed using the SPSS version 20.0 statistical package. Continuous data are presented using medians and interquartile ranges while categorical data are presented in frequencies and percentages. Early Infant Diagnosis (EID) was defined as infant access to or enrollment into HIV DNA-PCR testing before or at 6 weeks of birth.

EID (dichotomized) was analyzed using logistic regression to explore potential risk factors for not achieving early diagnosis. The models were adjusted for potential predictors including demographics, maternal age, ART status, residence, place of birth and maternal health condition. All variables in the univariate analysis with $P$ value of less than 0.2 were entered into multivariable logistic regression models. The odds ratio and $95 \%$ confidence intervals were also constructed along with their corresponding P values. The Kaplan-Meier method was used to estimate time-to-infant diagnostic test (i.e. time from birth to first enrollment for HIV DNA-PCR testing using DBS).

\section{Results}

A total of 266 mother-infant pairs were included in the analysis. The majority of women, 224 (84\%), were from urban areas, $48 \%$ had no formal education and $24 \%$ were divorced. The mean age of the women was 28 years (standard deviation: 5 years). Forty percent were already on HAART at the time of birth, but $22 \%$ of the mothers were diagnoses with HIV at or after the time of labor (Table 1). Overall, $82 \%$ of women who gave birth at home did not receive ARV prophylaxis at labor compared to $18 \%$ and $36 \%$ of women who gave birth at government and private clinics respectively (P value $<0.001$ ). In addition, $80 \%$ of infants born at home did not receive ARV prophylaxis immediately at birth compared to $10 \%$ and $32 \%$ of infants born at government and private facilities (P value $<0.001$ ).

\subsection{Early Infant Diagnosis and Follow-Up}

Of the 266 HIV-exposed infants identified from the 6 health facilities, only 109 (41\%) successfully had blood taken for HIV DNA-PCR testing on or before 6 weeks after delivery. The mean age at infant diagnosis was 120 days (95\% CI: 108 - 133 days) (Figure 1). The median turnaround time between blood draw for DNA-PCR testing to delivery of a test result to the respective health facility was 36 days (95\% CI: 33 - 40 days). A total of 35 (13.4\%) infants were diagnosed HIV positive; 17(6.5\%) were determined to be positive based on an HIV antibody test record. Thirty percent of women were lost to follow-up before they received their infant's HIV test results (Table 2).

Among the 109 infants who had the DBS test for HIV by 6 weeks, 77 (71\%) were born at the government hospital. A total of 58 (54\%) of the mothers whose infant achieved early diagnosis knew their HIV status prior to the current pregnancy and $83 \%$ were enrolled in the adult HIV clinic prior to delivery. In addition, out of those who accessed EID, $82.5 \%$ of infants were given ARV prophylaxis at birth and $75.2 \%$ of the women had received ARV prophylaxis during labor. 
Table 1. Baseline characteristics of the women-infant pairs.

\begin{tabular}{|c|c|c|}
\hline Characteristics & Response & Frequency (percent) \\
\hline \multirow{3}{*}{ Maternal age (years) } & $\leq 20$ & $15(5.7 \%)$ \\
\hline & $21-30$ & $185(69.8 \%)$ \\
\hline & $\geq 31$ & 65 (24.5\%) \\
\hline \multirow{4}{*}{ Educational status (grades attended) } & Uneducated & 125 (47.7\%) \\
\hline & Primary education (1 - 8) & $71(27.2 \%)$ \\
\hline & Secondary education & $52(19.8 \%)$ \\
\hline & College and above & $14(5.3 \%)$ \\
\hline \multirow{2}{*}{ Residence } & Urban & $224(84.2 \%)$ \\
\hline & Rural & $42(15.8 \%)$ \\
\hline \multirow{5}{*}{ Occupation } & Housewife (no outside work) & $120(45.8 \%)$ \\
\hline & Government employee & $23(8.7 \%)$ \\
\hline & Daily laborer & 88 (33.6\%) \\
\hline & Merchant & 17 (6.5\%) \\
\hline & Local drinking seller & $14(5.4 \%)$ \\
\hline \multirow{5}{*}{ Marital status } & Single & $19(7.3 \%)$ \\
\hline & Married & 149 (56.8\%) \\
\hline & Divorced & $61(23.3 \%)$ \\
\hline & Widowed & $24(9.2 \%)$ \\
\hline & Separated & $9(3.4 \%)$ \\
\hline \multirow{2}{*}{ Parity } & Primiparous & 67 (25.7\%) \\
\hline & multiparous & $194(74.3 \%)$ \\
\hline \multirow{4}{*}{ HIV infection identified } & Before the current pregnancy & $110(42.3 \%)$ \\
\hline & During the current pregnancy & $94(36.2 \%)$ \\
\hline & On labor/delivery & $26(10.0 \%)$ \\
\hline & During postnatal & $30(11.5 \%)$ \\
\hline \multirow{3}{*}{ Maternal ARV during pregnancy } & HAART & 105 (40.1\%) \\
\hline & Prophylaxis & 60 (22.9\%) \\
\hline & none & 97 (37.0\%) \\
\hline \multirow{2}{*}{ ANC follow-up } & Yes & 191 (75.7\%) \\
\hline & No & $61(24.3 \%)$ \\
\hline \multirow{3}{*}{ Place of delivery } & Home & 103 (39.0\%) \\
\hline & Government institution & $134(50.7 \%)$ \\
\hline & Private clinics/hospitals & 27 (10.3\%) \\
\hline \multirow{2}{*}{ Maternal ARV during labor } & Yes & 146 (55.3\%) \\
\hline & No & $118(44.7 \%)$ \\
\hline \multirow{2}{*}{ Infant ARV at delivery } & Yes & 159 (60.5\%) \\
\hline & No & 104 (39.5\%) \\
\hline \multirow{2}{*}{ Mothers health condition } & Alive & 247 (92.8\%) \\
\hline & Died & $19(7.2 \%)$ \\
\hline
\end{tabular}




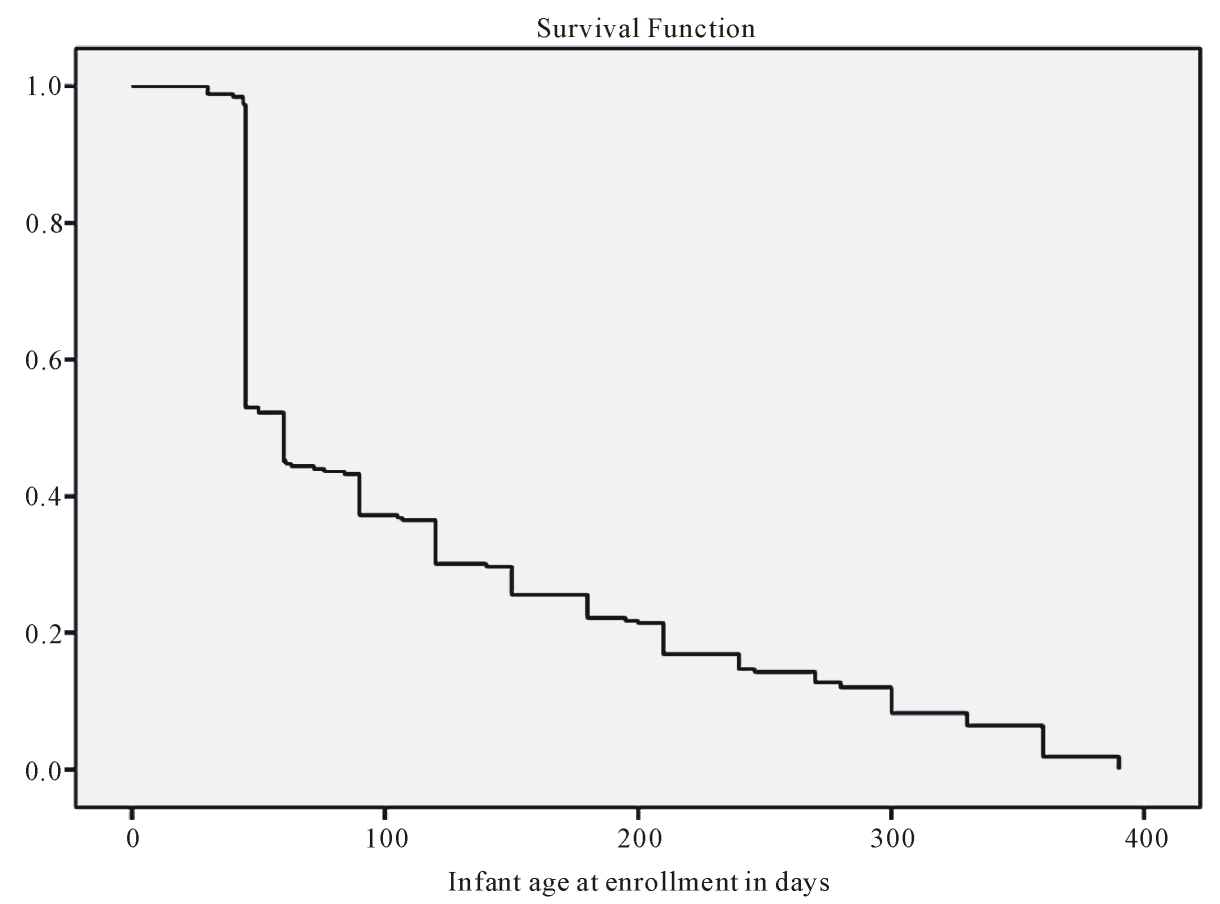

Figure 1. Kaplan Meier curve showing time from birth to blood collection for HIV DNA PCR in HIV-exposed infants (The median time from birth to blood collection for DBS was 60 days).

Table 2. Early infant diagnosis (EID, defined as blood draw before 45 days) and median time to process DBS samples and laboratory test.

\begin{tabular}{cc}
\hline Steps in the HIV diagnosis process & Number (percent) \\
\hline Number of infants with successful EID of HIV & $109 / 266$ (41\%) \\
Number of infants lost to follow-up prior to receiving HIV test results & $89 / 266$ (34\%) \\
Infants diagnosed positive for HIV & $37 / 266(13.2 \%)$ \\
Median age from birth to blood draw for HIV DNA-PCR test & 60 days [95\% CI; 47 - 73] \\
Median $\quad$ time $\quad$ from blood drawn to sample receipt by the central testing lab & 7 days [IQR: 4 - 13 days] \\
Median time from sample arrival at the central lab to test performance & 20 days [IQR: 14 - 35 days] \\
Median time between blood draw to result recipient by the respective health facility & 36 days [IQR: 25 - 49 days] \\
The mean age from birth to recipient of HIV DNA-PCR test result & 159 days [IQR: 81 - 215 days]
\end{tabular}

\subsection{Predictors of Accessing EID}

Table 3 shows the analysis of predictors of achieving early infant diagnosis. From the univariate analysis, six variables were significantly associated with an increased odds of achieving EID: 1) mothers or 2) infants who received ARV prophylaxis during labor or at delivery, 3) women who had ANC, 4) women who received ARV intervention (HAART or prophylaxis) during pregnancy, 5) women gave birth at government facility and 6) women who received their HIV diagnosis before or during the current pregnancy. Other variables that were tested but found not to be significantly associated with EID in univariate analyses included: maternal age, residence, occupation, educational status, parity, maternal health condition and marital status.

The multivariate logistic regression analysis of factors associated with EID demonstrated that mothers who were on HAART or had received PMTCT prophylaxis during pregnancy were more likely to successfully complete EID compared to those without these factors (odds ratios 3.4 and 3.7 respectively). Compared to being 
Table 3. Univariate and multivariate logistic regression analysis of the predictors of early infant diagnosis (HIV DNA-PCR performed at or before 45 days).

\begin{tabular}{|c|c|c|c|c|c|}
\hline \multirow{2}{*}{ Factors } & \multicolumn{2}{|c|}{$\begin{array}{l}\text { Infant HIV DNA-PCR at or } \\
\text { before } 45 \text { days }\end{array}$} & \multicolumn{2}{|c|}{ Odds Ratio (OR) (95\% C.I) } & \multirow{2}{*}{ P-Value } \\
\hline & Yes & No & Unadjusted OR & Adjusted OR & \\
\hline \multicolumn{6}{|l|}{ Prenatal care (ANC) } \\
\hline Yes & $92(89.3 \%)$ & $11(10.7 \%)$ & $4.2(2.0-8.6)$ & \multirow[b]{2}{*}{$1.7(0.7-4.3)$} & \multirow[b]{2}{*}{0.154} \\
\hline No & $99(66.4 \%)$ & $50(33.6 \%)$ & 1.0 & & \\
\hline \multicolumn{6}{|l|}{ ARV during pregnancy } \\
\hline HAART & $56(52.4 \%)$ & 49 (31.6\%) & $5.0(2.6-9.5)$ & $3.4(1.5-7.3)$ & \multirow{3}{*}{0.002} \\
\hline Prophylaxis & $33(30.8 \%)$ & 27 (17.5\%) & $5.4(2.6-11.0)$ & $3.7(1.5$ - 8.7) & \\
\hline None & $18(16.8 \%)$ & 79 (50.9\%) & 1.0 & 1.0 & \\
\hline \multicolumn{6}{|l|}{ Place of birth } \\
\hline Home & $25(22.9 \%)$ & $78(50.4 \%)$ & 1.0 & 1.0 & \multirow{3}{*}{$<0.001$} \\
\hline Private clinic & $7(6.5 \%)$ & 20 (12.9\%) & $1.1(0.4-2.8)$ & $0.5(0.2-1.7)$ & \\
\hline Government health facility & 77 (70.6\%) & $57(36.7 \%)$ & $4.2(2.4-7.4)$ & $2.9(1.6-5.5)$ & \\
\hline \multicolumn{6}{|l|}{ Time mother get HIV diagnosed } \\
\hline Before pregnancy & $58(53.7 \%)$ & $52(34.2 \%)$ & $7.2(2.3-22.2)$ & \multirow{4}{*}{$*$} & \multirow{4}{*}{$<0.001$} \\
\hline During pregnancy & $40(37.0 \%)$ & $54(35.5 \%)$ & $4.8(1.5-14.8)$ & & \\
\hline At labor/birth & $6(5.6 \%)$ & $20(13.2 \%)$ & $1.9(0.5-7.8)$ & & \\
\hline Postnatal & $4(3.7 \%)$ & $26(17.1 \%)$ & 1.0 & & \\
\hline \multicolumn{6}{|l|}{ Mother prophylaxis at labor } \\
\hline Yes & $82(75.2 \%)$ & $64(41.3)$ & $4.32(2.52-7.45)$ & * & \multirow{2}{*}{$<0.001$} \\
\hline No & $27(24.8 \%)$ & 91 (58.7\%) & 1.0 & & \\
\hline \multicolumn{6}{|l|}{ Infant prophylaxis at birth } \\
\hline Yes & $90(82.6 \%)$ & $69(44.8 \%)$ & $5.83(3.2-10.5)$ & * & \multirow{2}{*}{$<0.001$} \\
\hline No & $19(17.4 \%)$ & $85(55.2 \%)$ & 1.0 & & \\
\hline
\end{tabular}

$\mathrm{CI}=$ Confidence interval; ANC = Antenatal Care; $\mathrm{OR}=$ Odds Ratio; ${ }^{*}=$ variables not entered in the multivariable model due to significant correlation with other variables.

born at home, infants delivered at a government facility were almost three times more likely to have early infant diagnosis (odds ratio: 3.0, 95\% CI: 1.6 - 5.5). Three significant variables from the univariate analysis were excluded due to multiple colinearity (Table 3 ).

The median time from birth to sample collection for HIV DNA-PCR (infant HIV diagnosis) was 60 days (95\% CI: 47 - 73); (Figure 1). Since many women brought their infants in for testing at the customary post-natal visit, 6 weeks after birth, many visits occurred at 45 days. Women who received some form of ARV, either HAART or prophylaxis, brought their infants in for HIV-DNA PCR testing significantly earlier (median 45 days) than whose who received no form of ARV (median 150 days, $\mathrm{P}<0.001$; Figure 2). Table 2 shows the median time to process the DBS samples and the laboratory tests.

\section{Discussion}

Our study highlights the problem of delayed initiation and completion of HIV testing for infants born to HIVinfected mothers in northern Ethiopia. In fact, the infants at highest risk for HIV transmission, defined by their 


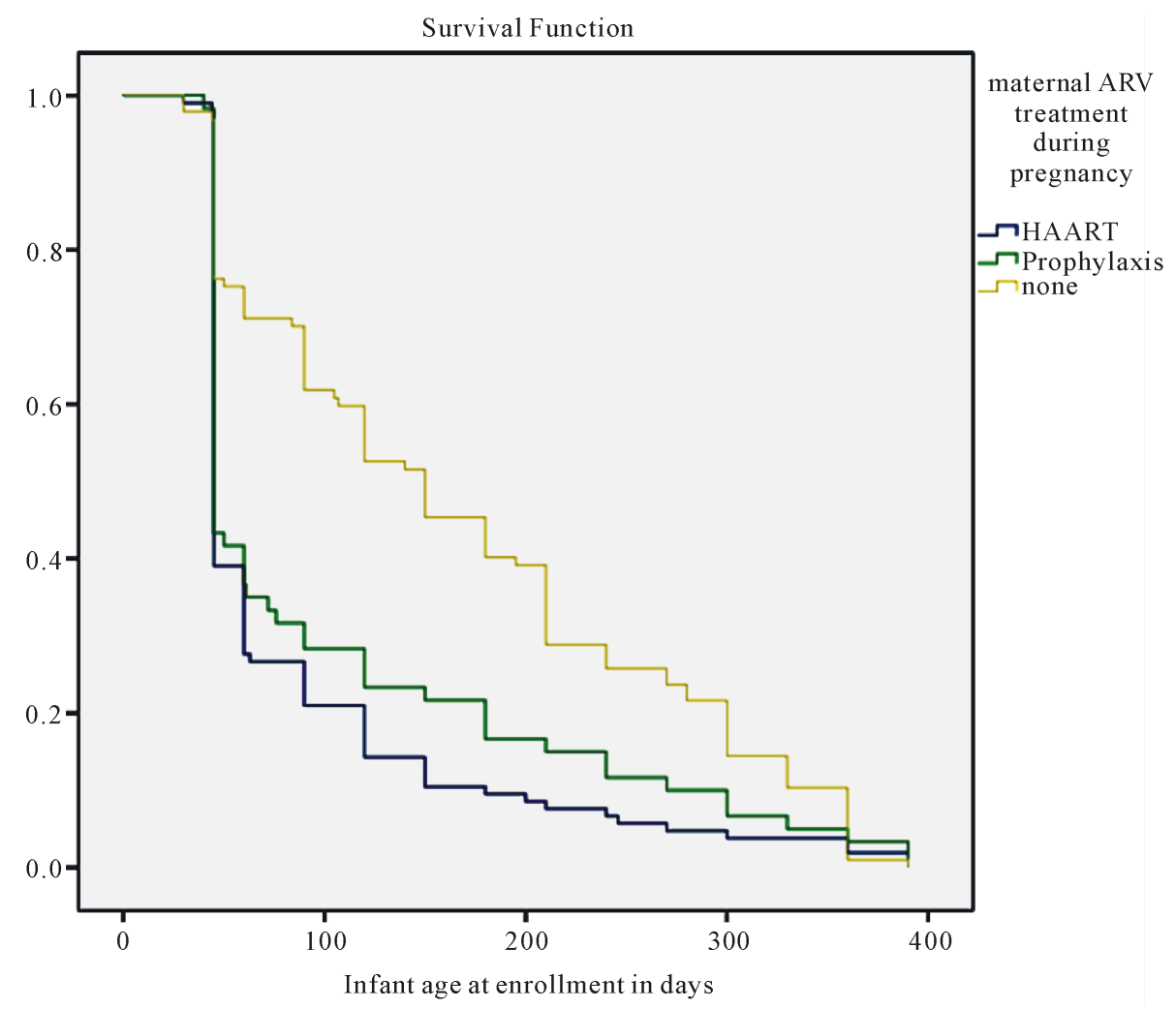

Figure 2. Kaplan Meier curves showing time from birth to blood collection for HIV DNA PCR in HIV-exposed infants by maternal ARV intervention status.

mothers not receiving any form of ARV prevention or treatment during pregnancy, had the greatest time to collection of HIV DNA test specimens (Figure 2). Several institutional and logistical challenges delay early HIV testing: the infant must be brought by a caregiver to a healthcare facility, the healthcare providers must offer DBS collection and the test should be performed without delays. [3]. Here, we describe the obstacles to achieving rapid HIV DNA-PCR testing for exposed infants, which is the first and essential step to enable the infected infants to receive life-saving ART care. We found both maternal and health system factors contributed to delaying the receipt of HIV test results: mothers/caregivers did not bring their exposed infant for testing at or before 6 weeks of birth and there was delays in sample collection, sample transport to the regional laboratory center, performance of the HIV test and return of the test result to the clinic and patient. All these factors would hinder enrollment of the infant into HIV care when needed. These delays are shown by Table 4 and Figure 3.

Overall, only $41 \%$ of infants had samples collected for HIV DNA-PCR testing at or before 6 weeks of life, the benchmark for achieving EID. The median time between blood draw for DBS to result recipient by the respective health facility was 36 days (IQR: 25 - 49 days). These findings are consistent with other studies from Kenya and Mozambique [8] [17] [18] but lower than study report from Cameroon and Malawi [9] [19].

The dynamics between place of delivery and PMTCT interventions are major challenges to early infant diagnosis. Mothers who delivered at government health institutions had the highest rate of early HIV DNA-PCR testing compared to deliveries which took place at home. Most mothers who gave birth at home did not receive ARV prophylaxis during labor and the infants were not given ARV prophylaxis immediately on delivery. In addition, mothers who delivered at private health facilities were less likely to bring their infants for early HIV testing.

Thirty seven percent of women in this study failed to take any form of ARV to prevent HIV transmission to their infants and the lack of ARV use was associated with delays in bringing the infant for early HIV diagnosis. Thus, not only would there be greater chance for HIV transmission, but infected infants would not be recognized and treated early; the combination would ultimately result in greater infant HIV morbidity and mortality. Fortunately, educational status, residence and marital status did not remain statistically significant association with 
Table 4. Factors contributed for delay in early infant diagnosis related to mother and processing of sample and laboratory.

\begin{tabular}{|c|c|}
\hline Delays & Contributing factors \\
\hline Delay one ${ }^{*}$ & Mothers delay in early return or not bringing the infants \\
\hline Delay two ${ }^{\#}$ & $\begin{array}{l}\text { Delay in sending the sample for DBS-PCR, Delay of the courier/postage system. Reported reasons: limit number } \\
\text { (minimum number of samples) for courier, poor cooperation of postal offices, and loss of samples on the way of } \\
\text { transport and the need to resend the sample twice. }\end{array}$ \\
\hline Delay three ${ }^{\#}$ & $\begin{array}{l}\text { Delay in laboratory processing in the regional laboratory center, Delay in resending the result or delay of the } \\
\text { courier/postage system. Reported reasons: light interruption at the central/regional laboratory, DBS testing Lab } \\
\text { chemicals use processing (under sampling), oversampling/overloading. }\end{array}$ \\
\hline Delay four ${ }^{*}$ & $\begin{array}{l}\text { Delay of the mothers/caregivers in returning to receive the test result, delay in the health professional early enrolling } \\
\text { the infants into care. Reasons reported: lack of reminding the mothers as most mothers forget the appointment, loss } \\
\text { of the mothers and/or death of the mothers/parents. }\end{array}$ \\
\hline
\end{tabular}

*Clients delay; ${ }^{*}$ Health system delay.

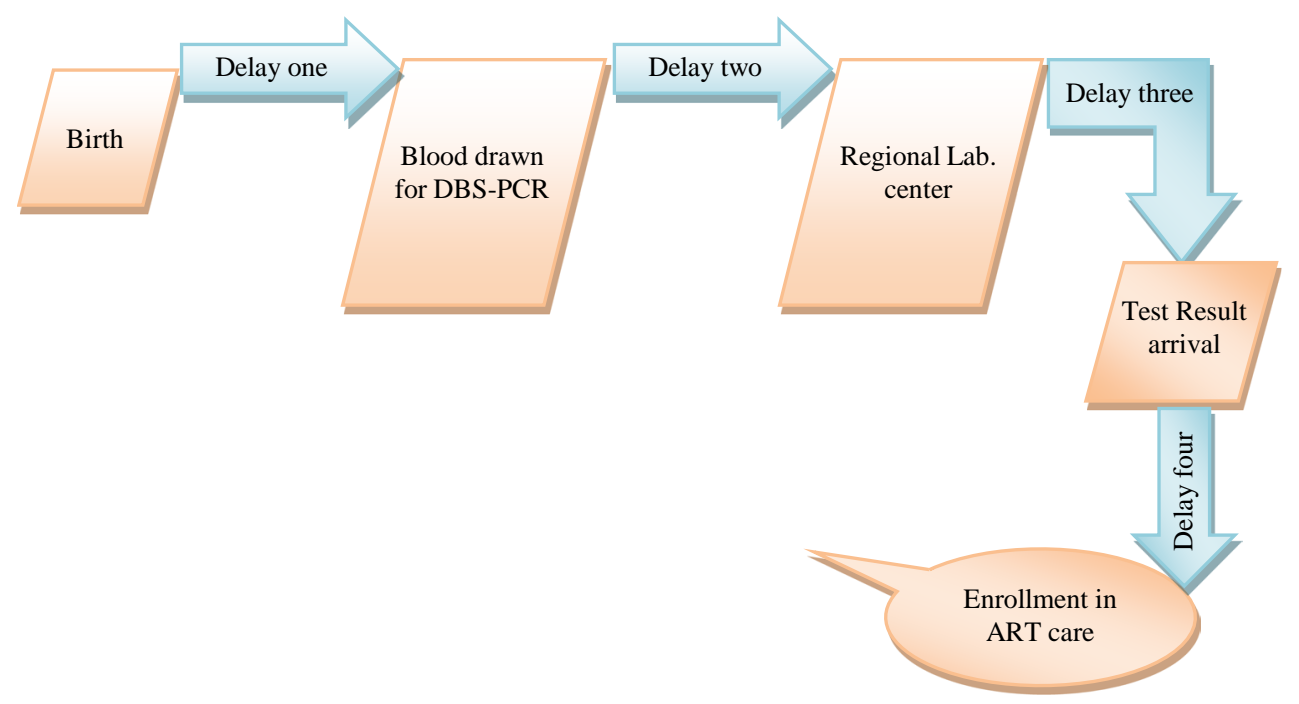

Figure 3. Diagram showing delay in early infant diagnosis and contributing factors.

EID, but prenatal care improved rates of EID. These findings corroborates those studies from Mozambique [18] and Malawi [20].

This study is limited by the retrospective, chart review nature of the design. In addition, the diagnosis of HIV infection of the infants was based both on HIV DNA-PCR and HIV antibody tests as recorded in the infants chart. However we were not able to verify that the date of antibody test was more than six months after birth and had to presume that the infant HIV antibody tests were done according to guidelines. Despite the limitations, these results emphasize the importance of conducting future prospective studies.

\section{Conclusions and Recommendation}

Three of five HIV-infected women did not bring their children for early infant HIV diagnosis. In addition to delays in blood collection, infant HIV diagnosis was further delayed by sample transportation, processing and result delivery. Both patient and health service factors require attention.

The main factors associated with delay in early infant diagnosis were location of delivery as well as lack of prenatal care and maternal use of ARV intervention. The first step to rectify these problems would be to increase maternal HIV testing during pregnancy which would then enable referral to PMTCT programs. Special attention should be given to strengthen services for women who choose to deliver at home. The finding that rates of EID were lower in women delivering at private clinics was surprising and should be addressed by greater HIV training for health care providers both during their primary health educational programs and as part of ongoing postgraduate education. Our group has emphasized augmenting the HIV-specific content of the curricula of under- 
graduate and postgraduate training of medical and ancillary healthcare providers as part of our UCSD PEPFAR program, but clearly more work is needed. Further, integrated maternal HIV testing, maternal ARV access and infant prophylaxis with postpartum advice for infant testing would increase rates of early infant diagnosis.

Medical records could be used to track lack of maternal follow-up in order to further reduce missing HIV diagnoses. Scheduling EID appointments to coincide with routine visits, i.e. first infant vaccination could eliminate the need for additional visits. In addition, reduction in test turnaround time, from sample collection, to laboratory, to the return of test results, is urgently required. Alternative methods to deliver test results to the clinic should be considered, such as use of email and telephone, instead of depending only on couriers.

\section{Acknowledgements}

We would like to thank the district health offices and health centers/hospitals for allowing us to conduct the study and access data, and the University of Gondar for ethical review. We are grateful to the study participants, data collection assistants and the hospital and health centers data managers.

This work was supported in part by University of Gondar Institute of Public Health and a grant from the HIV Research Trust and NIAID grants: AI 064086 (K24 to RH); AI 069432 (UCSD ACTU); and AI 36214 (CFAR Clinical Investigation and Biostatistics Core).

\section{Competing Interests}

The authors declare that there are no competing interests.

\section{Authors' Contribution}

BK: conceived and proposed the study, was involved in data collection and analyzed and drafted the manuscript. AG: revised the proposal and study design and was involved in initial data analysis. RH, SJ and SS: were involved in data cleaning, analysis and fully revised and edited the final manuscript.

\section{References}

[1] World Health Organization (2010) Antiretroviral Therapy for HIV Infection in Infants and Children: Recommendations for a Public Health Approach. http://www.who.int/hiv/pub/paediatric/infants2010/en/index.html

[2] UNAIDS (2012) Regional Fact Sheet. http://www.unaids.org/en/media/unaids/contentassets/documents/epidemiology/2012/gr2012/2012_FS_regional_ssa_en.pdf

[3] Ciaranello, A.L., Park, J.-E., Ramirez-Avila, L., Freedberg, K.A., Walensky, R.P. and Leroy, V. (2011) Early Infant HIV-1 Diagnosis Programs in Resource Limited Settings: Opportunities for Improved Outcomes and More Cost-Effective Interventions. BMC Medicine, 9, 59. http://dx.doi.org/10.1186/1741-7015-9-59

[4] World Health Organization. Report of the WHO Technical Reference Group Pediatric HIV ART/Care Guideline Group Meeting. http://www.who.int/hiv/pub/paediatric/WHO_Paediatric_ART_guideline_rev_mreport_2008.pdf

[5] Wamalwa, D., Benki-Nugent, S., Langat, A., Tapia, K., Ngugi, E., Slyker, J.A., Richardson, B.A. and John-Stewart, G.C. (2012) Survival Benefit of Early Infant Antiretroviral Therapy Is Compromised When Diagnosis Is Delayed. The Pediatric Infectious Disease Journal, 31, 729-731. http://dx.doi.org/10.1097/INF.0b013e3182587796

[6] World Health Organization (2010) WHO Recommendations on the Diagnosis of HIV Infection in Infants and Children. World Health Organization, Geneva.

[7] Mirkuzie, A.H., Hinderaker, S.G., Sisay, M.M., Moland, K.M. and Mørkve, O. (2011) Current Status of Medication Adherence and Infant Follow up in the Prevention of Mother to Child HIV Transmission Programme in Addis Ababa: A Cohort Study. Journal of the International AIDS Society, 14, 50. http://dx.doi.org/10.1186/1758-2652-14-50

[8] Hassan, A.S., Sakwa, E.M., Nabwera, H.M., Taegtmeyer, M.M., Kimutai, R.M., Sanders, E.J., Awuondo, K.K., Mutinda, M.N., Molyneux, C.S. and Berkley, J.A. (2012) Dynamics and Constraints of Early Infant Diagnosis of HIV Infection in Rural Kenya. AIDS and Behavior, 16, 5-12. http://dx.doi.org/10.1007/s10461-010-9877-7

[9] Tejiokem, M.C., Faye, A., Penda, I.C., Guemkam, G., Ndongo, F.A., Chewa, G.L., Rekacewicz, C., Rousset, D., Kfutwah, A., Boisier, P., et al. (2011) Feasibility of Early Infant Diagnosis of HIV in Resource-Limited Settings: The ANRS 12140-PEDIACAM Study in Cameroon. PLoS ONE, 6. http://dx.doi.org/10.1371/journal.pone.0021840

[10] Nuwagaba-Biribonwoha, H., Werq-Semo, B., Abdallah, A., Cunningham, A., Gamaliel, J.G., Mtunga, S., Nankabirwa, V., Malisa, I., Gonzalez, L.F., Massambu, C., et al. (2010) Introducing a Multi-Site Program for Early Diagnosis of HIV Infection among HIV-Exposed Infants in Tanzania. BMC Pediatrics, 10, 44. 
[11] HAPCO/FMOH (2007) Guidelines for Prevention of Mother-to-Child Transmission of HIV in Ethiopia. Federal HIV/ AIDS Prevention and Control Office/Federal Ministry of Health, Addis Ababa.

[12] Central Statistical Agency (Ethiopia) and ICF International (2012) Ethiopia Demographic and Health Survey 2011. Central Statistical Agency (Ethiopia) and ICF International, Addis Ababa and Calverton.

[13] Federal HAPCO (2010) Annual Performance Report of Multisectoral HIV/AIDS Response 2002 E.C. (2009/2010). Federal HIV/AIDS Prevention and Control Office, Addis Ababa.

[14] Mirkuzie, A.H., Hinderaker, S.G. and Mørkve, O. (2010) Promising Outcomes of a National Programme for the Prevention of Mother-to-Child HIV Transmission in Addis Ababa: A Retrospective Study. BMC Health Services Research, 10, 267.

[15] Nigatu, T. and Woldegebriel, Y. (2011) Analysis of the Prevention of Mother-to-Child Transmission (PMTCT) Service Utilization in Ethiopia: 2006-2010. Reproductive Health, 8, 6. http://dx.doi.org/10.1186/1742-4755-8-6

[16] Federal HIV/AIDS Prevention and Control Office/Federal Ministry of Health (2007) Guidelines for Paediatric HIV/ AIDS Care and Treatment in Ethiopia. Addis Ababa.

[17] Ciampa, P.J., Tique, J.A., Jumá, N., Sidat, M., Moon, T.D., Rothman, R.L. and Vermund, S.H. (2012) Addressing Poor Retention of Infants Exposed to HIV: A Quality Improvement Study in Rural Mozambique. Journal of Acquired Immune Deficiency Syndromes, 60, e46-e52. http://dx.doi.org/10.1097/QAI.0b013e31824c0267

[18] Cook, R.E., Ciampa, P.J., Sidat, M., Blevins, M., Burlison, J., Davidson, M.A., Arroz, J.A., Vergara, A.E., Vermund, S.H. and Moon, T.D. (2011) Predictors of Successful Early Infant Diagnosis of HIV in a Rural District Hospital in Zambézia, Mozambique. Journal of Acquired Immune Deficiency Syndromes, 56, e104-e109.

[19] Dube, Q., Dow, A., Chirambo, C., Lebov, J., Tenthani, L., Moore, M., Heydermana, R.S. and Rieb, A.V. (2012) Implementing Early Infant Diagnosis of HIV Infection at the Primary Care Level: Experiences and Challenges in Malawi. Bulletin of the World Health Organization, 90, 699-704.

[20] Jamieson, D.J., Chasela, C.S., Hudgens, M.G., King, C.C., Kourtis, A.P., Kayira, D., Hosseinipour, M.C., Kamwendo, D.D., Ellington, S.R., Wiener, Jr., B., et al. (2012) Maternal and Infant Antiretroviral Regimens to Prevent Postnatal HIV-1 Transmission: 48-Week Follow-Up of the BAN Randomized Controlled Trial. The Lancet, 379, 2449-2458. http://dx.doi.org/10.1016/S0140-6736(12)60321-3 
Scientific Research Publishing (SCIRP) is one of the largest Open Access journal publishers. It is currently publishing more than 200 open access, online, peer-reviewed journals covering a wide range of academic disciplines. SCIRP serves the worldwide academic communities and contributes to the progress and application of science with its publication.

Other selected journals from SCIRP are listed as below. Submit your manuscript to us via either submit@scirp.org or Online Submission Portal.
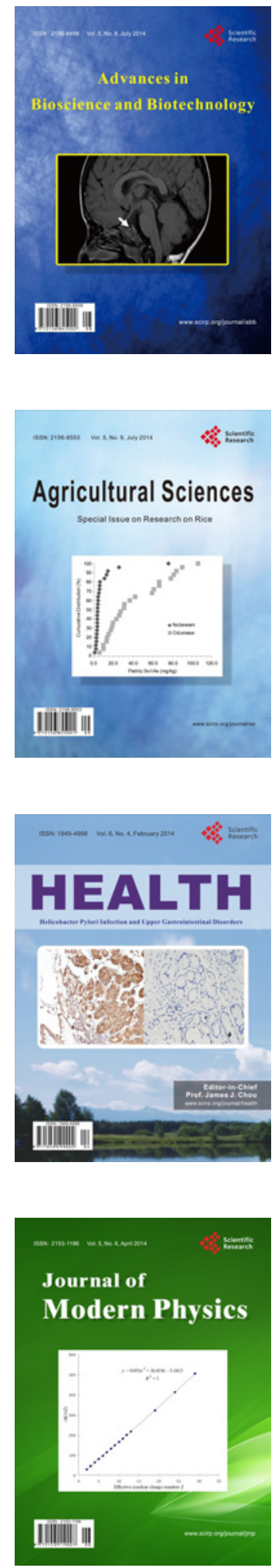
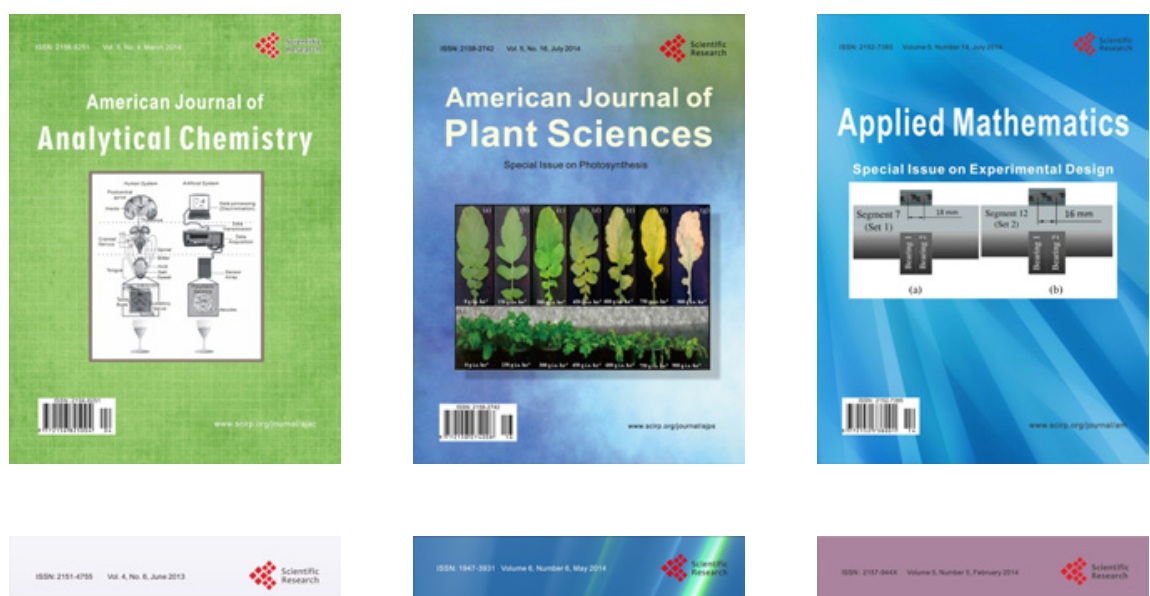

Creative Education
